\title{
Functional complementation of a yeast knockout strain by Schistosoma mansoni Rho1 GTPase in the presence of caffeine, an agent that affects mutants defective in the protein kinase $C$ signal transduction pathway
}

\author{
Pedro HN de Aguiar, Débora N Santos, Francisco P Lobo, Túlio M Santos, \\ Andréa M Macedo, Sérgio DJ Pena, Carlos R Machado, Glória R Franco ${ }^{+}$
}

Laboratório de Genética Bioquímica, Departamento de Bioquímica e Imunologia, Instituto de Ciências Biológicas, Universidade Federal de Minas Gerais. Av. Antônio Carlos 6627, 31270-901 Belo Horizonte, MG, Brasil

In a previous study, the Schistosoma mansoni Rhol protein was able to complement Rhol null mutant Saccharomyces cerevisiae cells at restrictive temperatures and under osmotic stress (low calcium concentration) better than the human homologue (RhoA). It is known that under osmotic stress, the S. cerevisiae Rhol triggers two distinct pathways: activation of the membrane 1,3- $\beta$-glucan synthase enzymatic complex and activation of the protein kinase C1 signal transduction pathway, promoting the transcription of response genes. In the present work the SmRhol protein and its mutants smrho $1^{\mathrm{E} 97 \mathrm{P}}, \mathrm{smrho}^{\mathrm{L} 101 \mathrm{~T}}$, and $\mathrm{smrho} 1^{\mathrm{E} 97 \mathrm{P}, \mathrm{L} 101 \mathrm{~T}}$ were used to try to clarify the basis for the differential complementation of Rhol knockout yeast strain by the human and S. mansoni genes. Experiments of functional complementation in the presence of caffeine and in the presence of the osmotic regulator sorbitol were conducted. SmRhol and its mutants showed a differential complementation of the yeast cells in the presence of caffeine, since smrho $1^{\mathrm{E} 97 \mathrm{P}}$ and $\mathrm{smrho} \mathrm{1}^{\mathrm{E} 97 \mathrm{P}, \mathrm{L} 101 \mathrm{~T}}$ mutants showed a delay in the growth when compared to the yeast complemented with the wild type SmRho1. However, in the presence of sorbitol and caffeine the wild type SmRho1 and mutants showed a similar complementation phenotype, as they allowed yeast growth in all caffeine concentrations tested.

Key words: Schistosoma mansoni - SmRho1 - protein kinase C1 - caffeine - Saccharomyces cerevisiae

The digenetic trematode, Schistosoma mansoni, is the causative agent of schistosomiasis, a disease that is still one of the most prevalent parasitic infections around the world (Engels et al. 2002). The understanding of parasite biology, mechanisms of drug resistance and antigenic variation that determine escape from the host immune system are the main reasons for studying parasites genomes. Therefore, $S$. mansoni transcriptome projects have contributed to the discovery of thousands of new genes in this parasite (Verjovski-Almeida et al. 2004). On the other hand, there are only $1242 \mathrm{~S}$. mansoni known protein sequences in contrast to 207,887 nucleotide sequences, according to the NCBI taxonomy database in February 20th 2006 (http://www.ncbi.nlm.nih.gov/entrez/ query.fcgi? $\mathrm{db}=$ Taxonomy), showing the importance of the characterization of novel genes.

The retention of $S$. mansoni eggs in the liver triggers inflammatory reactions that eventually may lead to liver fibrosis, the most serious pathological lesion of the disease (Dunne et al. 1995). A possible way to prevent these symptoms could be to inhibit egg laying. Several lines of evidence suggest the involvement of low-molecular weight GTP-binding proteins (LMWGPs) of the Ras su-

Financial support: CNPq, Capes, Fapemig ${ }^{+}$Corresponding author: gfranco@icb.ufmg.br Received 25 May 2006

Accepted 26 June 2006 perfamily in the maturation process and egg production of S. mansoni female worms (Schussler et al. 1997, VandeWaa et al. 1989). Thus, this superfamily of LMWGPs might represent an interesting target for the development of new anti-schistosomiasis drugs.

The Rho GTPase gene (SmRhol) from S. mansoni, encodes a 193 amino acids protein that is highly homologous to the Rho-type 1 LMWGPs of several species, has been previously characterized by Santos et al. (2002). The SmRhol gene was able to complement a $S$. cerevisiae Rhol null mutant strain even under temperature $\left(37^{\circ} \mathrm{C}\right)$ and osmotic (300 $\left.\mathrm{mM} \mathrm{CaCl}_{2}\right)$ stress conditions, contrasting with the human RhoA GTPase that was not able to provide complementation in such conditions (Qadota et al. 1994). The $\alpha 3$-helix loop7 had been previously defined by Qadota et al. (1994) as the region of the human RHOA protein responsible for its inability to complement yeast rhol null mutants at the conditions described above. After comparison of this region of the amino acid sequences from S. cerevisiae Rho1 (ScRho1), Candida albicans Rho1, human RhoA, and SmRho1, Santos et al. (2002) identified the proline 96 and threonine 100 residues of the human RhoA as the most probable determinants of the complementation differences between the human and worm genes. Three SmRhol point mutants (smrho ${ }^{E 97 P}$, smrho $1^{L 101 T}$, and smrho ${ }^{E 97 P, L 101 T}$ ) were generated by site directed mutagenesis and the conditional lethality phenotype at high temperature was reproduced in all mutants. This confirmed the importance of proline 96 and threonine 100 for the temperature-sensitive phenotype seen in 
yeast Rhol null mutant complemented by the human RhoA GTPase, and provided strong evidence that the related amino acid positions (Gln101 and Ile105) in the ScRho1 GTPase are indeed important for regulation of the cell wall synthesis performed by this protein in yeast.

The ScRhol protein participates in two classic pathways to perform its cellular functions in response to osmotic stress: activation of the $1,3-\beta$-glucan synthase enzyme complex for cell wall synthesis and remodeling and activation of the protein kinase $\mathrm{C}$ (PKC1) signal transduction pathway, which promotes the transcription of osmotic stress response genes (Sekiya-Kawasaki et al. 2002, Mager $\&$ Siderius 2002). In the present work we performed cell growth experiments in the presence of caffeine, known to affect mutants defective in the PKC1 signal transduction pathway by an unknown mechanism (Schmitz et al. 2002a), and sorbitol, an osmotic stabilizer, to investigate the differential behavior of the $S$. cerevisiae strains complemented with the three parasite mutants or the wild type SmRhol proteins. Our results suggest that the different functions performed by the Rho GTPases are related to the presence of certain amino acid residues at determined positions in the protein, and that evolutionary amino acid substitutions at such positions may modulate functional specificity in different species.

\section{MATERIALS AND METHODS}

In this work yeast cells from the CMY strain (MAT? ade $2 \Delta::$ hisG? his $3 \Delta 200$ met $15 \Delta 0$ trp $1 \Delta 63$ ura3 $\Delta 0$ leu $2 \Delta 0$ lys $2 \Delta 0$ Drho1::HIS3 pYEDP(SMRHO1)) transformed with the following constructions: pYTS(scrho1), pYTS(smrho 1), pYTS(smrho1 ${ }^{\text {E9P }) \text {, }}$ pYTS(smrho1 $\left.{ }^{\text {L101T }}\right), p Y T S\left(\right.$ smrho1 ${ }^{\text {E97P, L101T) }}$ ) and $p Y T S$ were used (Santos et al. 2002). Yeast cells were grown in YPD rich medium (1\% yeast extract, $2 \%$ peptone, $2 \%$ glucose $\mathrm{pH} 7.0$, or either $2 \%$ galactose for CMY cells containing only the pYTS empty vector). For yeast cells survival experiments, cells were grown overnight at $30^{\circ} \mathrm{C}$ in YPD rich medium, and after counting in a Neubauer chamber, a total of $10^{4}$ cells were inoculated in YPD medium supplemented with different concentrations of caffeine and sorbitol as indicated. After $36 \mathrm{~h}$ of incubation at $30^{\circ} \mathrm{C}$, optical density (OD) at $660 \mathrm{~nm}$ of the yeast cells was measured in the Spectra Max 340 (Molecular Devices) spectrophotometer.

\section{RESULTS}

In order to analyze the consequences of point mutations in the ability of SmRho1 to phenotypically complement an yeast Rhol null mutant, we utilized haploid yeast cells complemented by the $S$. mansoni homologous gene cloned into the pYEDP vector. The Rhol deletion is lethal for the yeast and only those cells complemented with ScRhol or with an heterologous Rho protein are able to grow (Santos et al. 2002). The pYEDP vector contains a GAL10 promoter inducing gene overexpression in the presence of galactose. The yeast cells were transformed with a second construction in the pYTS plasmid, which contains the $S c R h o 1$ promoter driving a low constitutive gene expression. The cDNAs from ScRhol, SmRhol and its mutants cited above were cloned into the pYTS plasmid
(Santos et al. 2002). Hence, in the presence of galactose, SmRhol cloned into the pYEDP vector is overexpressed, and in medium containing glucose, SmRhol overexpression is not induced and the yeast cells are complemented by the expression of the cDNAs cloned into the pYTS plasmid. The experiments described here were performed in the presence of glucose in order to obtain the expression of pYTS constructions.

Yeast cells survival curves were produced with the $S$. cerevisiae Rhol null mutant strain complemented with ScRhol, SmRhol, and its mutants in the presence of glucose and different concentrations of caffeine. Fig. 1A shows that the yeast strains complemented with the ScRhol and the SmRhol wild type protein are able to grow similarly to the wild type cells (non $S c R h o 1$ deleted) at all caffeine concentrations tested. The yeast cells carrying the smrho $1^{\text {E97P }}$ and the smrho1 ${ }^{\text {E97P/LI01T }}$ genes present a less efficient functional complementation above $2 \mathrm{mM}$ caffeine, when compared to strains complemented with the S. mansoni wild type protein and the L101T mutant protein. The yeast strain complemented with the smrho $1^{L 101 T}$ mutant only shows significant differences on its growth in caffeine concentrations above $5 \mathrm{mM}$. This result points out the importance of the glutamate residue for the protein function. However, in the same conditions with the addition of sorbitol, all yeast strains complemented with SmRhol protein and its mutants presented a growth rate comparable to the yeast cells comple-
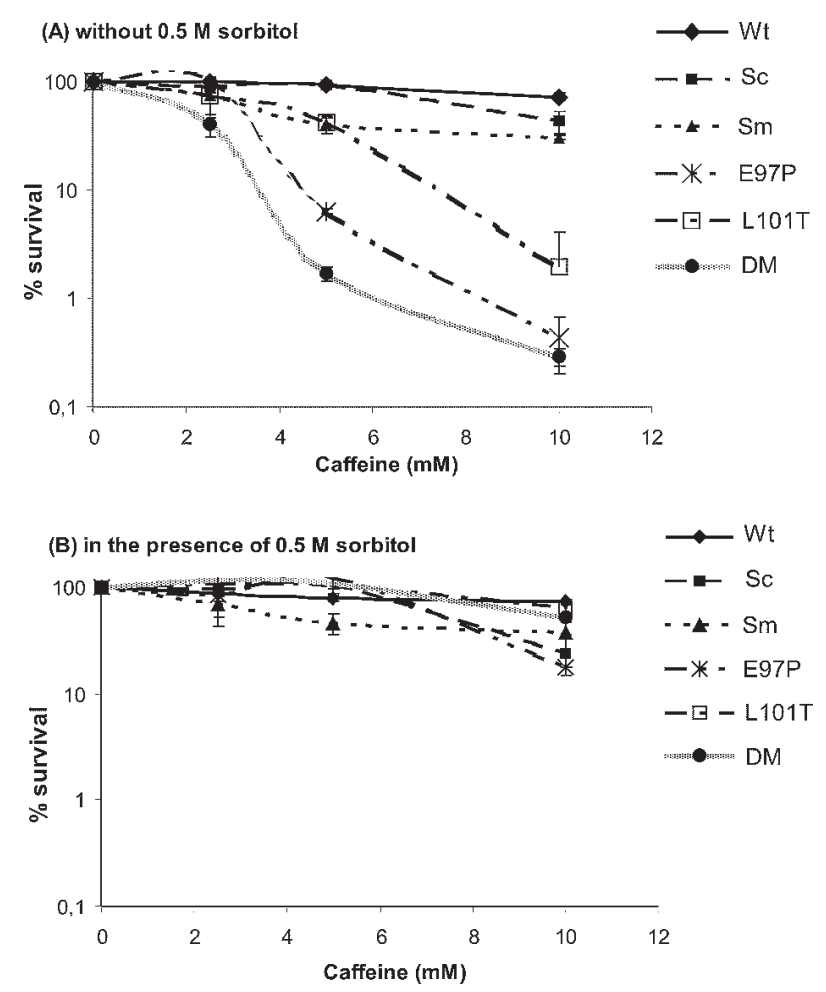

Fig. 1: survival curves of the wild type Saccharomyces cerevisiae cells (Wt), CMY yeast strain transformed with $p Y T S /$ scrhol $(\mathrm{Sc})$, pYTS/smrho1 (Sm), pYTS/smrho1 ${ }^{\text {E97P }}$ (E97P), pYTS/smrho1 L101T (L101T), and pYTS/smrho1 ${ }^{\text {E97P, L101T }}$ (DM). Yeast cells were grown for $36 \mathrm{~h}$ at $30^{\circ} \mathrm{C}$ in YPD rich medium supplemented with caffeine in the indicated concentrations without sorbitol (A) and in the presence of $0.5 \mathrm{M}$ sorbitol as osmotic stabilizer (B). 
mented with the ScRhol protein or the wild type cells (Fig. 1B), indicating that sorbitol, an osmotic stabilizer, restores yeast cells normal growth.

\section{DISCUSSION}

In this present study we investigated the differential growth of the $S$. cerevisiae Rhol null mutant strains complemented with the mutants or the wild type SmRho1 proteins through cell growth experiments in the presence of caffeine, an agent that was reported to inhibit growth of yeast strains containing deletions on the PKC1-mediated MAP kinase pathway (Costigan et al. 1992, Schmitz et al. 2002a), and sorbitol, an osmotic stabilizer.

The manner by which caffeine acts on yeast cells is not well understood. At first, it was thought to inhibit phosphodiesterase activity in the cAMP/Ras pathway of glucose signaling (Riley \& Barclay 1990). However, double deletions in the genes encoding the two isoenzymes of phosphodiesterase in yeast (Dpde1, Dpde2) showed no modification of the Mpk1p phosphorilation status, a protein which is situated downstream of PKC1 on its signaling pathway (Martin et al. 2000). On the other hand, Schmitz et al. (2002b) reported that a Rho5 (known to downregulate PKC activity) deleted yeast strain shows an increased activity of PKC-dependent signal transduction pathway and therefore an increased resistance to caffeine. Most importantly, the deletion of the HR1A domain in the amino-terminal region of $\mathrm{PKC} 1$ produced a strain that is sensitive to caffeine but is capable of activating the MAP kinase cascade (Schmitz et al. 2002a).

The $S$. cerevisiae $\mathrm{Rho1}$ is a regulatory protein required for 1,3- $\beta$-glucan synthase enzyme complex activity (Qadota et al. 1996, Firon et al. 2004), for activation of protein kinase $\mathrm{C}$ and the cell integrity pathway (Heinisch et al. 1999), for cell polarization and bud emergence (Mack et al. 1996) and for progression in cell cycle and exocitosis (Drgonova et al. 1999). This protein was reported to interact with $\mathrm{PKC} 1$ through its $\mathrm{C} 1$ domain, activating the MAP kinase pathway, and through its HR1 domain, leading to the activation of other PKC1-specific events (Schmitz et al. 2002a).

We confirmed in this work that the SmRhol homology (62\% identity) to $S$. cerevisiae Rho1 is sufficient to complement yeast Rho1 GTPase functions, as previously observed. This complementation was adequate to permit survival and growth even under osmotic stress conditions (10 mM caffeine; Fig. 1A). Thus, the $S$. mansoni Rhol protein can apparently perform all the functions described above in the yeast cells. On the other hand, Santos et al. (2002) reported phenotypic alterations, such as cell cycle arrest and cell enlargement caused by SmRho 1 overexpression in the yeast Rhol null mutant strain. It was suggested then that the $S$. mansoni GTPase is not able to perform all the ScRhol gene functions probably due to defective molecular interactions of the SmRho1 with some of the distinct Rho1 regulatory and effector proteins, caused by amino acid differences existing among the heterologous and native GTPases.

Moreover, we demonstrated that the SmRhol mutant proteins are not able to fully complement the yeast Rhol null mutant strain when compared to the SmRhol wild type and ScRhol proteins. The smrhol ${ }^{\text {E97P }}$, smrhol ${ }^{L 101 T}$, and smrho $1^{E 97 P, L 101 T}$ mutants growth under osmotic stress conditions (5-10 mM caffeine) confirmed the importance of proline 96 and threonine 100 for the osmotic stress sensitive phenotype seen in yeast Rhol null mutant complemented by the human RhoA GTPase. The main SmRho1 mutation responsible for the differential complementation phenotype observed for the yeast Rhol null mutant is the E97P amino acid change. Thus, the double mutant presented the most drastic effect onto the ability of cell wall synthesis by the yeast under osmotic stress, probably due to an important conformational alteration induced in the $\alpha 3$-helix. Nevertheless, the effect of replacing a leucine by a threonine at position 101 was less drastic than the E97P mutation. It is well-known that a proline can disrupt $\alpha$-helices in water soluble proteins (Barlow \& Thornton 1988, Kumar \& Bansal 1998) modifying the native structural conformation and compromising protein function and interactions. Threonine residues are also common in bend regions of twisted $\alpha$-helices (Kumar \& Bansal 1998). Thus, the threonine residue in the position 100 of human RhoA could produce a conformational difference that would have functional repercussions. However, in the presence of $0.5 \mathrm{M}$ sorbitol as osmotic stabilizer the caffeine-sensitive phenotype was suppressed (Fig. 1B), providing strong evidence that one of the functions performed by the $S$. cerevisiae Rho1 should be the maintenance of the yeast cell wall integrity.

The SmRhol mutants shared the same caffeine sensitive phenotype observed in an yeast strain deleted in the PKC1 HR1 domain (Schmitz et al. 2002a). This result suggests that the E97P and L101T mutations could be responsible for abrogating the Rho1-PKC1 HR1 domain interaction as depicted in Fig. 2. On the other hand, as $0.5 \mathrm{M}$ sorbitol restores the yeast cells normal growth, we could speculate that the mutations referred above could also interfere with the binding of SmRho1 to the $\mathrm{Cl}$ domain of

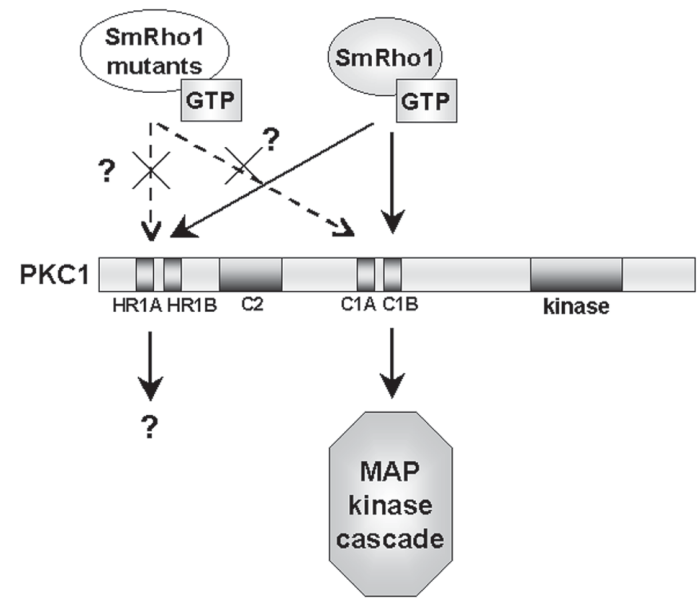

Fig. 2: hypothetical model for SmRhol interaction with the yeast (protein kinase $\mathrm{C} 1$ ). When activated through the binding of GTP, the SmRhol protein contacts both the $\mathrm{C} 1$ and HR1 domain of the yeast PKC1 protein, activating two distinct pathways. We propose that the SmRhol mutants could be unable to bind to one of these domains due to conformational changes caused by the amino acids replacements (modified from Schmitz et al. 2002). 
the yeast $\mathrm{PKC} 1$ protein, affecting the cell integrity pathway, represented in Fig. 2 as the MAP kinase cascade.

In conclusion, although the Rho family of proteins are small molecules that share a high degree of homology in different species, subtle amino acid differences in certain regions can determine their interaction specificity with the multiple regulatory and effector proteins of each organism (Saka et al. 2001). The human RhoA and SmRho1 have different structures and probably show different interactions, therefore, the SmRhol gene could be an important target for the development of new antischistosomiasis drugs.

\section{ACKNOWLEDGEMENTS}

To Neuza Antunes Rodrigues and Miriam Rodrigues for technical support.

\section{REFERENCES}

Barlow DJ, Thornton JM 1988. Helix geometry in proteins. $J$ Mol Biol 201: 601-619.

Costigan C, Gehrung S, Snyder M 1992. A synthetic lethal screen identifies SLK1, a novel protein kinase homolog implicated in yeast cell morphogenesis and cell growth. $\mathrm{Mol}$ Cell Biol 12: 1162-1178.

Drgonova J, Drgon T, Roh DH, Cabib E 1999. The GTP-binding protein $\mathrm{Rhol} p$ is required for cell cycle progression and polarization of the yeast cell. J Cell Biol 146: 373-387.

Dunne DW, Hagan P, Abath FGC 1995. Prospects for immunological control of schistosomiasis. The Lancet 345: 14881492.

Engels D, Chitsulo L, Montressor A, Savioli L 2002. The global epidemiological situation of schistosomiasis and new approaches to control and research. Acta Trop 82: 139-146.

Firon A, Lesage G, Bussey H 2004. Integrative studies put cell wall synthesis on the yeast functional map. Curr Opin Microbiol 7: 617-623.

Heinisch JJ, Lorberg A, Schmitz HP, Jacoby JJ 1999. The protein kinase C-mediated MAP kinase pathway involved in the maintenance of cellular integrity in Saccharomyces cerevisiae. Mol Microbiol 32: 671-680.

Kumar S, Bansal M 1998. Geometrical and sequence characteristics of $\alpha$-helices in globular proteins. Biophys J 75: 19351944.

Mack D, Nishimura K, Dennehey BK, Arbogast T, Parkinson J, Toh-e A, Pringle JR, Bender A, Matsui Y 1996. Identification of the bud emergence gene BEM4 and its interactions with Rho-type GTPases in Saccharomyces cerevisiae. Mol Cell Biol 16: 4387-4395.
Mager WH, Siderius M 2002. Novel insights into the osmotic stress response of yeast. FEMS Yeast Res 2: 251-257.

Martin H, Rodriguez-Pachon JM, Ruiz C, Nombela C, Molina M 2000. Regulatory mechanisms for modulation of signaling through the cell integrity Slt2-mediated pathway in Saccharomyces cerevisiae. J Biol Chem 275: 1511-1519.

Qadota H, Anraku Y, Botstein D, Ohya Y 1994. Conditional lethality of a yeast strain expressing human RHOA in place of RHO1. Proc Natl Acad Sci USA 91: 9317-9321.

Qadota H, Python CP, Inoue SB, Arisawa M, Anraku Y, Zheng Y 1996. Identification of yeast Rholp GTPase as a regulatory subunit of 1,3- $\beta$-glucan synthase. Science 272: 279281.

Riley BB, Barclay SL 1990. Conditions that alter intracellular cAMP levels affect expression of the cAMP phosphodiesterase gene in Dictyostelium. Proc Natl Acad Sci USA 87: 4746-4750.

Saka A, Abe M, Okano H, Minemura M, Qadota H, Utsugi T, Mino A, Tanaka K, Takai Y, Ohya Y 2001. Complementing yeast rho1 mutation groups with distinct functional defects. J Biol Chem 276: 46165-46171.

Santos TM, Machado CR, Franco GR, Pena SDJ 2002. Characterization and comparative functional analysis in yeast of a Schistosoma mansoni Rho1 GTPase gene. Mol Biochem Parasitol 125: 103-112.

Schmitz HP, Huppert S, Lorberg A, Heinisch JJ 2002b. Rho5p downregulates the yeast cell integrity pathway. J Cell Sci 115: 3139-3148.

Schmitz HP, Lorberg A, Heinisch JJ 2002a. Regulation of yeast protein kinase $\mathrm{C}$ activity by interaction with the small GTPase Rholp through its amino-terminal HR1 domain. Mol Microbiol 44: 829-840.

Schussler P, Grevelding CG, Kunz W 1997. Identification of Ras, MAP kinases, and a GAP protein in Schistosoma mansoni by immunoblotting and their putative involvement in male-female interaction. Parasitology 115: 629-634.

Sekiya-Kawasaki M, Abe M, Saka A, Watanabe D, Kono K, Minemura-Asakawa M, Ishihara S, Watanabe T, Ohya Y 2002. Dissection of upstream regulatory components of the Rholp effector, 1,3- $\beta$-glucan synthase, in Saccharomyces cerevisiae. Genetics 162: 663-676.

VandeWaa EA, Mills G, Chen GZ, Foster LA, Bennett JL 1989. Physiological role of HMG-CoA reductase in regulating egg production by Schistosoma mansoni. Am J Physiol 257: 641-645.

Verjovski-Almeida S, Leite LCC, Dias-Neto E, Menck CFM, Wilson RA 2004. Schistosome transcriptome: insights and perspectives for functional genomics. Trends Parasitol 20: 304-308. 